\title{
Morphological features of breast cancer circulating tumor cells in blood after physical and biological type of isolation
}

\footnotetext{
1 Department of Experimental Oncology, Institute of Oncology Ljubljana, Ljubljana, Slovenia

2 Faculty of Medicine, University of Ljubljana, Ljubljana, Slovenia

${ }^{3}$ Department of Medical Oncology, Institute of Oncology Ljubljana, Ljubljana, Slovenia

${ }^{4}$ Faculty of Health Sciences, University of Primorska, Izola, Slovenia.

${ }^{5}$ Department of Cytopathology, Institute of Oncology Ljubljana, Ljubljana, Slovenia
}

Tanja Jesenko ${ }^{1,2}$, Ziva Modic ${ }^{1,2}$, Cvetka Grasic Kuharr,3, Maja Cemazar ${ }^{1,4}$, Urska Matkovic ${ }^{1}$, Simona Miceska ${ }^{2,5}$, Jerneja Varl2,5, Anamarija Kuhar ${ }^{5}$ and Veronika Kloboves-Prevodnik ${ }^{2,5}$

Radiol Oncol 2021; 55(3): 292-304.

Received 5 July 2021

Accepted 9 July 2021

Correspondence to: Assoc. Prof. Veronika Kloboves-Prevodnik, M.D., Ph.D., Department of Cytopathology, Institute of Oncology Ljubljana, Slovenia, email:vkloboves@onko-i.si, and Maja Cemažar, Department of Experimental Oncology, Institute of Oncology Ljubljana, Slovenia. E-mail: mcemazar@onko-i.si

Disclosure: No potential conflicts of interest were disclosed.

This is an open access article under the CC BY-NC-ND license (http://creativecommons.org/licenses/by-nc-nd/4.0/).

Background. Circulating tumor cells (CTCS) have become an important biomarker in breast cancer. Different isolation tech-niques based on their biological or physical features were established. Currently, the most widely used methods for visualization after their separation are based on immunofluorescent staining, which does not provide the information on the morphology.

Materials and methods. The aim of this study was to evaluate how two different separation techniques affect cell morphology and to analyse cell morphology with techniques used in routine cytopathological laboratory. A direct side-by-side comparison of physical (Parsortix $\circledast$ ) and biological (MACS®) separation technique was performed.

Results. In the preclinical setting, both isolation techniques retained the viability and antigenic characteristics of MCF7 breast cancer cells. Some signs of degeneration such as cell swelling, cytoplasmic blebs, villous projections and vacuolization were observed. In metastatic breast cancer patient cohort, morphological features of isolated CTCs were dependent on the separation technique. After physical separation, CTCs with preserved cell morphology were detected. After biological separation the majority of the isolated CTCs were so degenerated that their identity was difficult to confirm.

Conclusions. Taken together, physical separation is a suitable technique for detection of CTCs with preserved cell morphology for the use in a routine cytopathological laboratory.

Key words: CTC; breast cancer; morphology; Parsortix®, MACS®; Giemsa

\section{Introduction}

Circulating tumor cells (CTCs) have become an important biomarker in breast cancer as they can provide critical information about disease progression and response to therapy. ${ }^{1,2}$ They represent an intermediate part of the metastatic cascade, therefore monitoring CTC levels in the blood has exceptional implications for the management of cancer patients. ${ }^{3}$ In the blood stream, CTCs are a heterogeneous cell population of tumor cells with different phenotypes. They show high level of epithelial-mesenchymal plasticity and can express an epithelial phenotype, mesenchymal phenotype or even a hybrid or partial epithelial/mesenchymal $(\mathrm{E} / \mathrm{M})$ phenotype in which cells express proteins of both phenotypes. ${ }^{4,5}$ 
Due to their heterogeneity, different isolation techniques from whole blood samples were established in order to separate them from the surrounding hematopoietic cells. Separation techniques can be based on CTC biological or physical properties. Biological separation techniques rely on the expression of cell markers and are usually based on epithelial cell markers positive selection or common leukocyte antigen CD45 negative selection. Currently the only FDA approved platform for enumeration of CTCs CELLSEARCH (Menarini Silicon Biosystems) is based on biological properties, which enables the detection of CTCs of epithelial origin (CD45-, EpCAM+, and cytokeratins $8,18+$, and/or 19+) in whole blood by fluorescent staining and imaging of cells that are pulled to a single focal depth by a magnetic force. ${ }^{6}$ An alternative technique that also relies on EpCAM positive selection and magnetic force is Magnetic Activated Cell Sorting (MACS $®$, Miltenyi Biotec). ${ }^{7}$ This technique was evaluated in our previous study for the isolation of CTCs in early breast cancer patients. ${ }^{8}$ It was demonstrated to be a simple and useful method for enrichment of EpCAM expressing cells in a preclinical study, however the isolated CTCs from early breast cancer patients were not morphologically preserved enough for their visualization by methods used in a routine cytopathology laboratory. The limitation of separation techniques based on biological properties is that only CTCs that express the epithelial cell markers can be detected. Due to this limitation, platforms exploiting physical properties such as cell size, density, electric charge and deformability are gaining more attention. With the Parsortix ${ }^{\circledR}$ system (ANGLE), CTCs are caught in the Parsortix ${ }^{\circledR}$ filtration cassette due to their larger size and lower compressibility than other blood components. ${ }^{9}$ The isolated cells are viable, intact and can be used for further in vitro experimentation and characterization. ${ }^{9}$

Currently, most methods for visualization of CTCs after separation from whole blood are usually based on staining with fluorescent antibodies and dyes. Therefore, these methods lack the information on the CTC morphology that is crucial for their identification by light microscopy which is still the gold standard in cytopathological and histological examination of tumor cells. The identification of CTCs by their morphology is challenging as these cells are often severely degenerated due to the combination of physical stress (shear forces), immune surveillance and the lack of growth factors in the blood stream.,10 Furthermore, separation methods could also induce some additional dam- age that can result in degeneration and cell death which change morphological features of CTCs and influence their proper identification. ${ }^{11}$ Probably for these reasons, data regarding basic CTC morphology in breast cancer are limited and thus the selection of the appropriate separation method is of outmost importance for proper cytopathological identification. ${ }^{11-14}$

There are few published data how separation techniques affect CTCs morphology. ${ }^{13-16}$ Therefore, we designed a study aimed to select the separation method that would allow the identification of CTCs based on their morphology that could be integrated in a routine cytopathology laboratory. The advantage of cytopathological analysis is that it is easily accessible in the clinical environment as cytopathologists are an integral part of cancer patient care. Two separation techniques were first evaluated in the preclinical setting by spiking of blood of healthy volunteers with MCF7 breast cancer cell line, which is the most studied human breast cancer cell line. ${ }^{17}$ The preclinical evaluation was followed by a prospective clinical trial in metastatic breast cancer patients with the primary objective being a side-by-side comparison of both separation techniques. The aims of this study were i) to evaluate how two different separation techniques affect cell morphology and ii) to analyze cell morphology with techniques used in routine cytopathological laboratory.

\section{Materials and methods}

\section{Ethic statement}

The study was conducted at the Institute of Oncology Ljubljana, Slovenia and was reviewed and approved by the Institutional Ethical Review Committee (ref. nb. ERID-KSOPKR-0071/2020) and National Medical Ethics Committee at the Slovenian Ministry of Health (ref. nb. 0120-1502019/4). All enrolled patients and healthy volunteers signed an informed consent. The study was conducted in accordance with the Declaration of Helsinki. Blood samples were collected during the patients' routine blood draw, which is a minimally invasive procedure.

\section{Patients and healthy volunteers}

Thirty-three patients were recruited in the study, however only 30 patients donated their blood samples, therefore their identification numbers are in the range $\mathrm{P} 1$ to $\mathrm{P} 33$. Three healthy volunteers were 
(A)

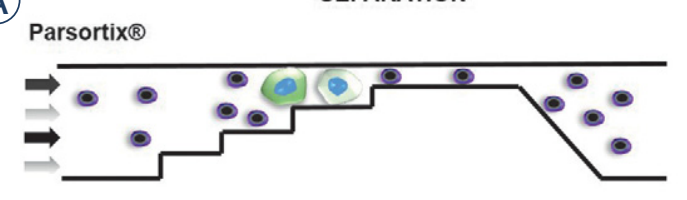

SEPARATION

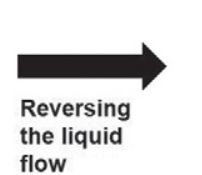

HARVESTING / ELUTION

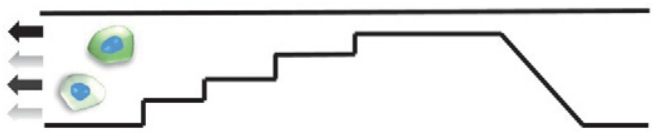

(B) $\operatorname{MACS} \circledast$

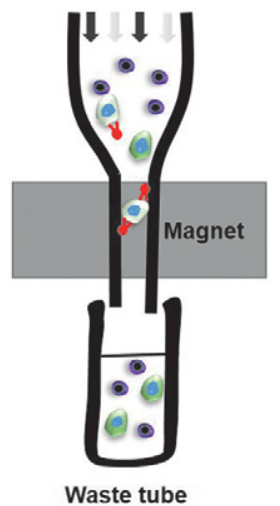

Mesenchymal CTC

Epithelial CTC (EрCAM+)

Magnetic bead conjugated

to EpCAM antibody

Blood cell
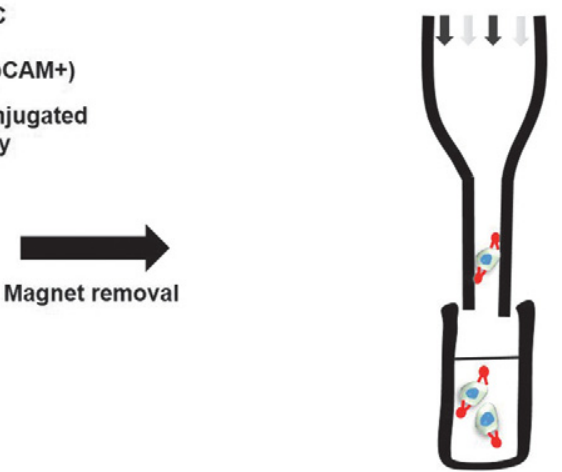

New collection tube

FIGURE 1. Schematic presentation of both separation techniques. (A) Parsortix(B) separation cassette contain a stepped structure, gradually narrowing in diameter until reaching a final gap of $6.5 \mu \mathrm{m}$, therefore all of the cells that are larger than $6.5 \mu \mathrm{m}$ are retained and isolated while all smaller cells continue to flow through the cassette into a waste container. The technique enables the isolation of CTCs with different phenotypes. After the whole sample has been processed, the liquid flow is reversed and CTCs can be harvested. (B) MACS® separation column uses magnetic beads covered with anti-epithelial cell adhesion molecule (EpCAM) antibodies for positive selection of CTCs with epithelial origin. When magnetic bead labeled CTCs are passed through a dense magnetic column, they are retained by a strong magnetic field. Other non-labeled cells are passed through the column into the waste tube. After the whole sample has been passed through the column, the column is removed from the magnet and retained CTCs can be eluted.

also included in the study. Each patient or healthy volunteer donated two blood samples in a $10 \mathrm{ml}$ EDTA collection tube (BD, Franklin Lakes, NJ, USA). The blood samples were processed within 1 $h$ after collection to ensure the highest viability of the CTCs. One tube was used for Parsortix ${ }^{\circledR}$ and the other for MACS® separation. After separation, cytological slides were prepared as described below.

\section{Spiking of MCF7 cell line}

Human epithelial breast cancer cell line MCF7 was obtained from ATCC (ATCC® HTB-22, ATCC, Manassas, VA, USA) and was cultured in Advanced MEM medium (Gibco, Thermo Fisher Scientific, Waltham, MA, USA) supplemented with 5\% fetal bovine serum (Gibco), GlutaMAX (100x, Gibco) and Penicillin-Streptomycin solution (Sigma Aldrich, Merck, Darmstadt, Germany). The cells were cultured in a $5 \% \mathrm{CO}_{2}$ humidified incubator at $37^{\circ} \mathrm{C}$ until they reached $80 \%$ confluence. Afterwards, the medium was removed, the cells were washed with phosphate buffered saline
(PBS) and detached from the surface with $0.25 \%$ trypsin/EDTA in Hank's buffer (Gibco). After collection, the cells were counted and $5 \times 10^{5}$ cells were spiked into each blood collection tube and separated by Parsortix ${ }^{\circledR}$ or MACS®. For the purpose of retaining control non-separated cells, the same amount of MCF7 cells was seeded into a 24-well ultra-low attachment plate and incubated in a 5\% $\mathrm{CO}_{2}$ humidified incubator at $37^{\circ} \mathrm{C}$ until the end of Parsortix ${ }^{\circledR}$ and MACS $®$ separation.

\section{Separation techniques}

Parsortix ${ }^{\circledR}$ separation was conducted by carefully following the manufacturer's instructions. Parsortix ${ }^{\circledR}$ separation cassette contains a stepped structure, gradually narrowing in diameter until reaching a final gap of $6.5 \mu \mathrm{m}$, therefore all of the cells that are larger than $6.5 \mu \mathrm{m}$ are retained and isolated, while all smaller cells continue to flow through the cassette into a waste container (Figure 1A). ${ }^{18}$ After the separation, retained cells were harvested into a $5 \mathrm{ml}$ plain red-top BD vacutainer tube without a pre-harvest flush and re- 
suspended in an in-house cell medium: $20 \%$ fetal bovine serum (Gibco), 5\% EDTA (Sigma Aldrich) in PBS. MACS® separation was performed as previously described (Figure 1B) and harvested in MACS® Whole Blood Column Elution Buffer ${ }^{8}$. MACS® separation is not limited by the cell size.

\section{Preparation of cytological slides}

For preclinical part of the study, three cytospins were prepared using a cytocentrifuge (Thermo Scientific Shandon Cytospin R 4 Cytocentrifuge, Waltham, MA, USA) by centrifugation at $700 \mathrm{rpm}$ for $4 \mathrm{~min}$ at room temperature (RT). The first cytospin was stained with Giemsa (Sigma Aldrich, Merck) for microscopic evaluation, the second one was fixed in Delaunay $(2500 \mathrm{~mL}$ acetone, $2500 \mathrm{~mL}$ absolute ethanol and 2,5 mL $1 \mathrm{M}$ trichloroacetic acid) and stained with Papanicolaou (PAP), which was subsequently stained for pancytokeratin AE1/ AE3. The third one was fixed in methanol for estrogen receptor immunocytochemical staining. For clinical part of the study two cytospins were made, one for Giemsa staining for morphological evaluation and the second for PAP staining and subsequent dual AE1/AE3 and vimentin immunocytochemical staining.

\section{Staining}

For Giemsa staining, the slide was air dried for at least 30 minutes at room temperature and stained with Giemsa. The second cytospin was fixed (Delaunay, 2 hours or overnight) and stained PAP using an automated stainer Leica Multistainer ST5020 (Leica Microsystems, Buffalo Grove, IL, United States).

Immunocytochemical staining of CK AE1/AE3 and estrogen receptor (ER) was performed with our routine immunocytochemical staining protocols. All protocols included manually performed endogenous peroxidase activity inhibition with $5 \% \mathrm{H}_{2} \mathrm{O}_{2}$-methanol solution for $10 \mathrm{~min}$ at RT. After the incubation was done, slides were washed once 1x PBS and once with Reaction Buffer (Ventana, Roche Diagnostics). The presence of the antigens was detected with iView detection kit on Ultra autostainer (Ventana, Roche Diagnostics). Enzymatic detection was accomplished when a streptavidin enzyme (streptavidin-HRP) conjugates with the biotin-bound secondary antibody. Chromogen was deposited by a reaction with hydrogen peroxide in the presence of diaminobenzidine (DAB) and copper sulfate, producing brown precipitate. External positive controls were used in all batches.

Immunocytochemical staining of the CK AE1/ AE3 and vimentin was performed as dual staining on the same PAP stained cytospin. The CK AE1/ AE3 staining was performed with anti CK monoclonal antibody (CK AE1/AE3, ref. nr. M3515, dilution 1:100, Agilent, Santa Clara, CA, United States. The vimentin immune staining was performed after the CK AE1/AE3 staining was done, with anti-vimentin antibody (V9, ref. nr. M0725, dilution 1:500, Agilent, Santa Clara, CA, United States). The presence of vimentin was detected with ultraView Universal Alkaline Phosphatase Red Detection Kit (Ventana, Roche Diagnostics), enzymatic detection was accomplished with an alkaline phosphatase and chromogen Fast Red, producing red precipitate.

For ER staining, methanol fixed cytospin was stained with anti ER monoclonal antibody (ER, ref. nr. NCL-L-ER-6F11, dilution 1:25, Novocastra, Leica Biosystems, United Kingdom). ER protocol included additional manual antigen retrieval step with previously boiled 1x TRIS-EDTA buffer solution ( $\mathrm{pH}$ ) for $3 \mathrm{~min}$.

\section{Evaluation of cytological slides}

Giemsa slides were evaluated by an experienced cytopathologist (VKP) and images of the cells on slides were captured with a DP72 CCD camera connected to a BX-51 microscope (Olympus, Hamburg, Germany. In the preclinical part, morphological features were quantified in 100 cells from each experimental group. Cell diameter, nuclear diameter and thickness of the cytoplasm were measured using ImageJ software. ${ }^{19}$ Other morphological features that were analyzed were cytoplasmic and nuclear chromasia, degeneration characteristics (cytoplasmic blebs, cytoplasmic villous projections and vacuolization), regularity of plasma membrane and nuclear membrane and chromatin features. These morphological features were quantified by counting the cells that displayed these characteristics. In the clinical part of the study, the slides were evaluated by the cytopathologist and CTCs were identified by their cytomorphological features. Based on their morphological appearance, they were categorized as morphologically "preserved" or "unpreserved". Criteria for the preserved CTCs were: cells with morphological features of malignancy such as large nuclei, high nuclear to cytoplasmic (N/C) ratio, scant cytoplasm, visible chromatin structure or presence of mitotic figures. Criteria for identifica- 
tion of morphologically unpreserved CTCs were: cells with morphological features of malignancy such as large nuclei, high N/C ratio, scant cytoplasm and loss of chromatin structure and nuclear membrane integrity. All of the identified CTCs were imaged and their diameter was measured.

\section{Flow cytometry}

Cell viability and type of cell death of MCF7 cells was determined by FITC Annexin V Apoptosis Detection Kit with 7-AAD (BioLegend, San Diego, CA, USA) according to manufacturer's instructions. Within 1 hour after separation, Annexin V and 7AAD were added to the cells and the measurements were performed on at least 100,000 cells per sample using FACSCanto II flow cytometer (BD Biosciences, San Jose, CA). Data were analyzed using FlowJo software.

\section{Statistical analysis}

The values in this study are represented by median with interquartile range, mean \pm standard error of the mean (SE) or mean with 95\% confidence interval and are defined in the figure legends. The patient's categorical characteristics were presented as frequencies and percentages. Age was presented as median and range. The comparison of means of more than two groups was statistically evaluated by one-way ANOVA followed by a Dunnett's multiple comparisons test. The comparison of medians of more than two groups was evaluated by Kruskal-Wallis one-way ANOVA followed by Dunn's multiple comparisons test. Fisher's exact test was used to determine the significance between two categorical variables. A P-value of $<0.05$ was considered to be statistically significant. A sample size (n) for each experiment is stated in the figure legend. For statistical analysis and preparation of graphs, GraphPad Prism 8 (La Jolla, CA, USA) was used.

\section{Results}

\section{Hydropic degeneration after Parsortix ${ }^{\circledR}$ and $\mathrm{MACS} \otimes$ separation}

In the first part of the study, the effect of both separation techniques on cell morphology was evaluated on Giemsa stained cytological slides of intact (non-separated) MCF7 cells vs. Parsortix ${ }^{\circledR}$ or MACS® isolated MCF7 cells. In comparison to the intact MCF7 cells (Figure 2A, No separation), the Parsortix® and MACS® separated MCF7 cells showed signs of cell swelling i.e. hydropic degeneration (Figure 2A, Parsortix $®$ and MACS®). Also high background of blood cells was observed after MACS $®$ separation protocol (Figure 2A, MACS®).

Preservation of antigenic epitopes for immunocytochemical staining was determined by immunocytochemical staining of AE1/AE3 cytokeratin and estrogen receptor, as these stainings are usually used in the cytopathological detection of breast cancer cells. Both separation techniques retained the epitopes for immunocytochemical staining. All MCF7 cells presented with strong cytoplasmic AE1/ AE3 cytokeratin positive staining (Figure 2B) in all experimental groups. Nuclear estrogen receptor staining was positive in around $70 \%$ of MCF7 cells either in non-separated cells or after Parsortix ${ }^{\circledR}$ or MACS® separation (Figure $2 \mathrm{C}$ ).

Cell swelling was quantified based on the measurements of cell diameter, nuclear diameter and thickness of the cytoplasm (Figure 2D). Both separation techniques induced similar statistically significant enlargement of the cell diameter (Figure 2E), nuclear diameter (Figure 2F) and thickness of the cytoplasm (Figure 2G). Due to the increase in the thickness of cytoplasm and size of nucleus, cytoplasmic staining was significantly altered. Dark basophilic staining was statistically significantly reduced after both separation techniques (Figure $2 \mathrm{H}$ ). Contrary, pale basophilic staining increased in the opposite manner, with MACS $\AA$ separated cells having the highest fraction of pale basophilic cytoplasmic staining (Figure 2H). The plasma membrane was intact after both separation techniques (Figure 2A). The nuclear features such as chromatin structure and nucleoli were preserved after both separation techniques (Figure 1A). Nuclear staining was mostly hyperchromatic (Figure 2A). Chromatin was mostly coarsely granular (Figure 2D). The nuclear membrane was intact with some irregularities observed after both separation techniques (Figure 2A).

\section{Degenerative cytoplasmic changes and cell viability after Parsortix ${ }^{\circledR}$ and $M A C S \circledR$ separation}

Increased fraction of cells with degenerative cytoplasmic changes were observed in Giemsa stained MCF7 cells after both separation techniques. Morphologically non-degenerated cells were presented with homogenous cytoplasm and intact smooth plasma membrane (Figure 3A). Degenerative changes that were observed more 

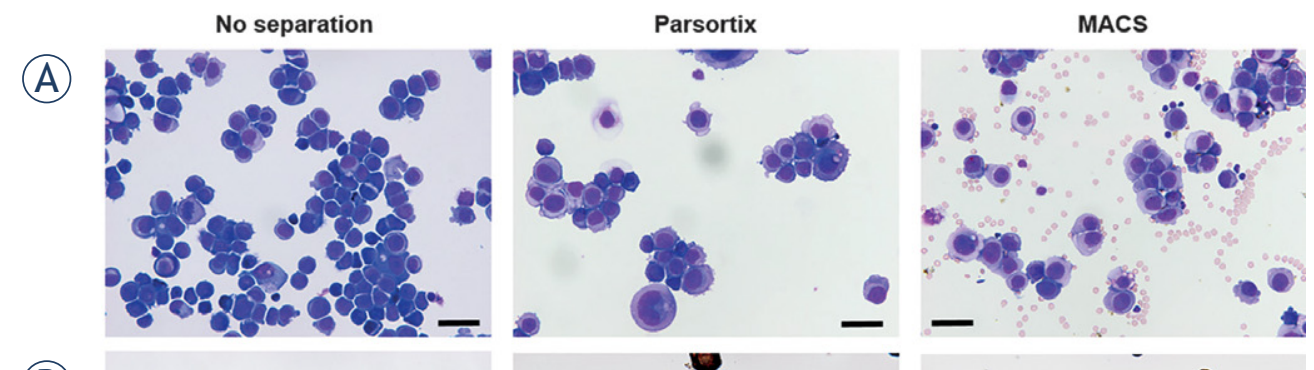

(B)

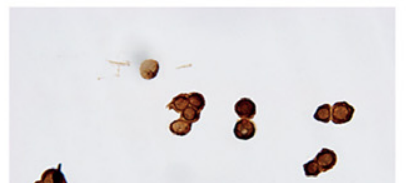

(C)
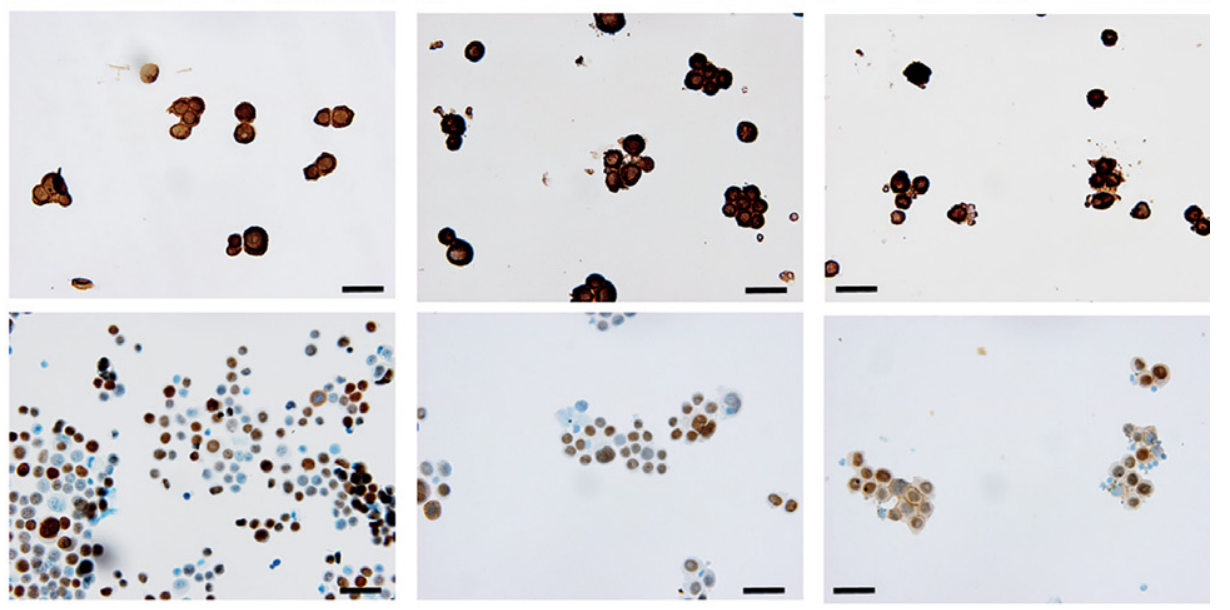

(D)

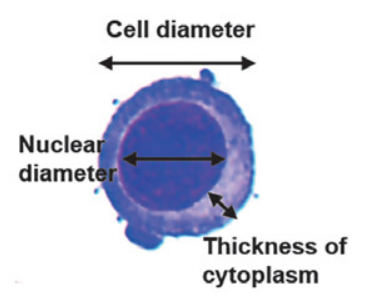

(E)
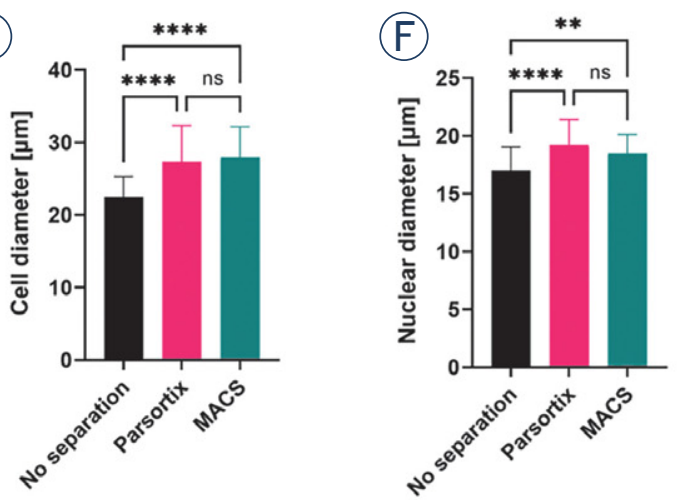

(G)
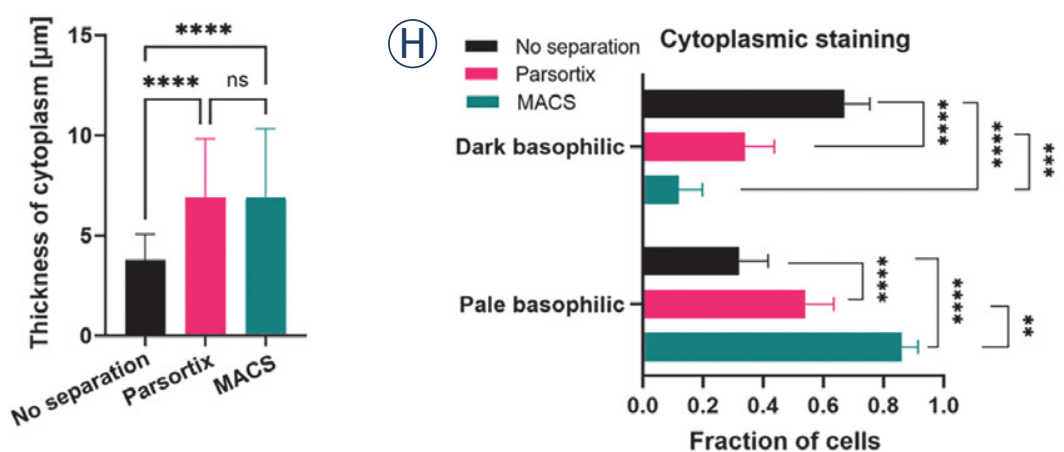

FIGURE 2. Parsortix ${ }^{\circledR}$ and $M A C S \AA$ separation techniques induced hydropic degeneration of cells. (A) Giemsa stained, (B) AEl/ AE3 cytokeratin stained and (C) estrogen receptor stained non-separated MCF7 cells (No separation) and separated MCF7 cells by Parsortix $\AA$ (Parsortix $\AA$ ) or MACS $\AA$ (MACS $\AA$ ). Scale bar represents $50 \mu \mathrm{m}$. Note that estrogen receptor stain is nuclear therefore cells appear smaller due to the poor contrasting of the cytoplasm. (D) Presentation of measured and evaluated cell sizes. Nonseparated and Parsortix ${ }^{\circledR}$ or MACS ${ }^{\circledR}$ separated $(E)$ cell diameter of, $n=100$ cells, $(F)$ nuclear diameter, $n=100$ cells and (G) thickness of cytoplasm, n=100 cells. (H) Cytoplasmic staining of non-separated MCF7 cells and Parsortix ${ }^{\circledR}$ or MACS $\AA$ separated cells, $n=$ 100 cells. Values in (E, F, G) represent median with interquartile range and $(\mathrm{H})$ mean with $95 \%$ confidence interval. Statistical significance was determined by Kruskal-Wallis one-way ANOVA followed by Dunn's multiple comparisons test for (E, F, G) and by Fisher's exact test for $\mathbf{( H )}$. 
(A)

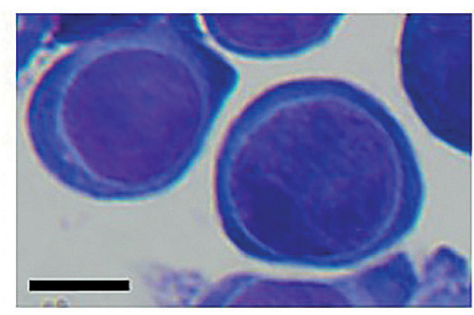

(B)

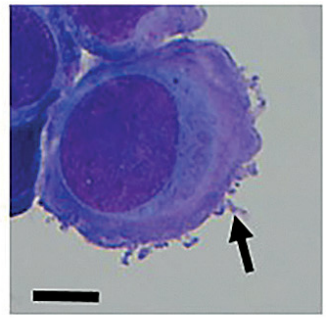

(C)

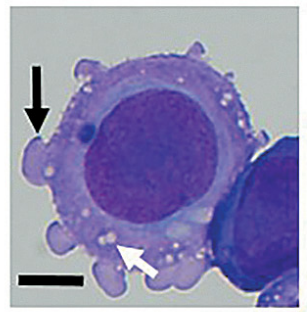

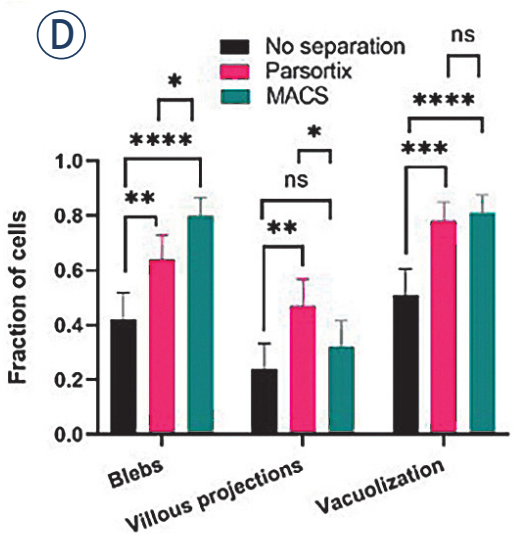

(E) No separation

Parsortix

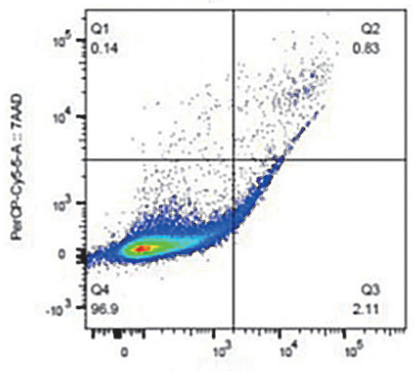

FITCA $=$ Amein

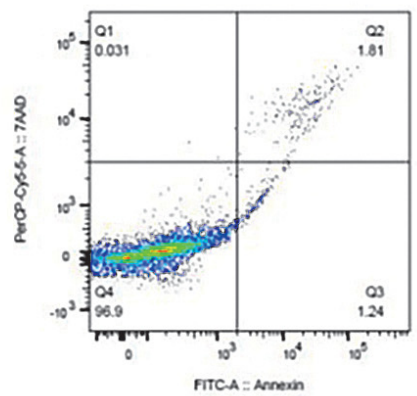

MACS

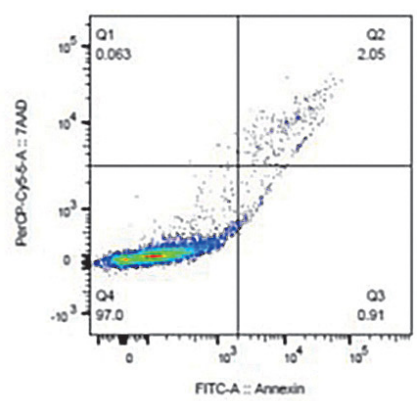

(F)

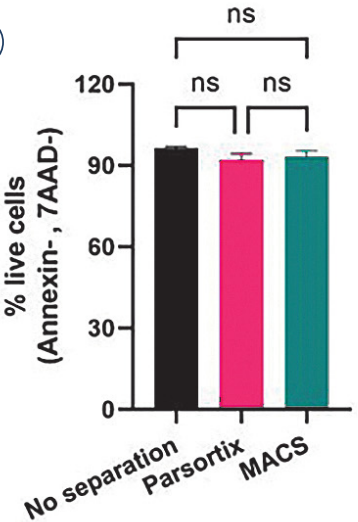

FIGURE 3. Parsortix ${ }^{\circledR}$ and $M A C S \AA$ separation techniques induced degenerative cytoplasmic changes but retained the viability of MCF7 cells. (A) Morphologically non-degenerated cells presented with homogenous cytoplasm and intact smooth plasma membrane. Scale bar represents 10 um. (B) Cytoplasmic villous projections as observed degenerative changes are indicated by black arrow. Scale represents $10 \mu \mathrm{m}$. (C) Membrane blebbing (indicated by black arrow) and cytoplasmic vacuolization (indicate by white arrow) as observed degenerative changes. Scale represents $10 \mu \mathrm{m}$. (D) Fraction of cells with villous projections, blebs or vacuolization. Values represent mean with $95 \%$ confidence interval, $n=100$ cells. Statistical significance was determined by Fisher's exact test. ${ }^{*}=p<0.05,{ }^{* *}=p<0.01,{ }^{* * *}=p<0.001,{ }^{* * * *}=p<0.0001$, ns = not significant. (E) Representative dot plots of Annexin $\checkmark$ and 7AAD staining in non-separated cells and Parsortix and MACS separated cells ( $F$ ) Percent of live cells (Annexin $V-$, 7AAD-), $n=3$. The values represent mean $\pm(S E), n=$ number of biological replicates. Statistical significance was determined by one-way ANOVA followed by a Dunnett's multiple comparisons test; ns- not significant.

frequently after both separation protocols were cytoplasmic villous projections (Figure 3B, black arrow), membrane blebbing (Figure $3 \mathrm{C}$, black arrow) and cytoplasmic vacuolization (Figure 3C, white arrow). Quantification of the observed changes demonstrated that MACS ${ }^{\circledR}$ separation induced the highest increase in the fraction of cells with cytoplasmic blebs (Figure 3D). Villous projections were more frequent in Parsortix ${ }^{\circledR}$ separated cells (Figure 3D). Increased fraction of cells with cytoplasmic vacuoles was observed after both separation techniques (Figure 3D).

However, although degenerative changes were observed after both separation techniques, the separated cells retained their viability. Flow cytometric analysis of Annexin V (apoptosis) and 7AAD (necrosis) demonstrated that MCF7 cells remained their viability after both separation techniques (Figure 3E). The quantification demonstrated that the percentage of live cells (Annexin V-, 7AAD-) remained unchanged following both separation techniques (Figure 3F). No significant changes were observed in the percentage of cells in early apoptosis (Annexin $\mathrm{V}+$, 7AAD), late apoptosis (Annexin $\mathrm{V}+, 7 \mathrm{AAD}+$ ) and necrosis (Annexin $\mathrm{V}-$, 7AAD+) among the groups (Figure 3E).

\section{Evaluation of both separation techniques for isolation of CTCs in metastatic breast cancer}

Both separation techniques were also evaluated in clinical setting in metastatic breast cancer patients. Patient characteristics are presented in Table 1. The median age of the patients was 58.1 years (min 39.5, $\max 79.3$ ).

CTCs were isolated side-by-side by Parsortix ${ }^{\circledR}$ and MACS® separation, each from $10 \mathrm{ml}$ of whole 
(A)

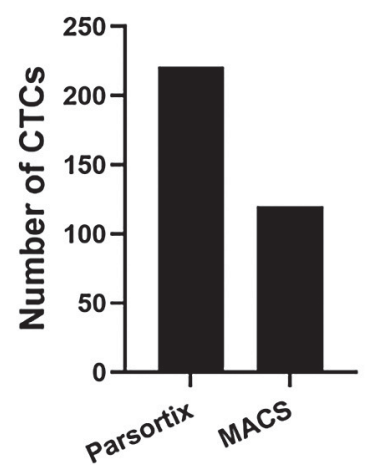

(D)

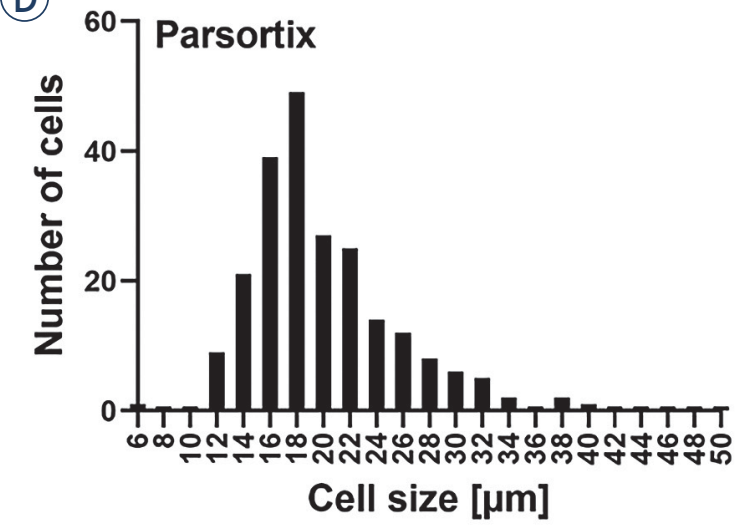

(B)

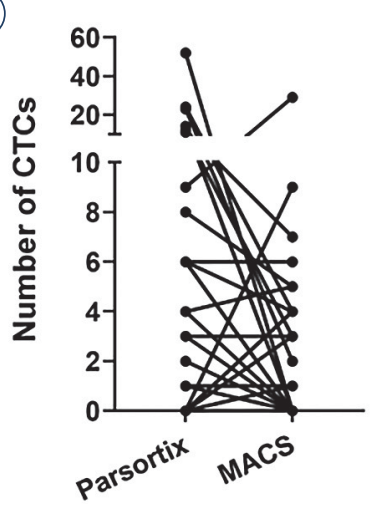

(C)

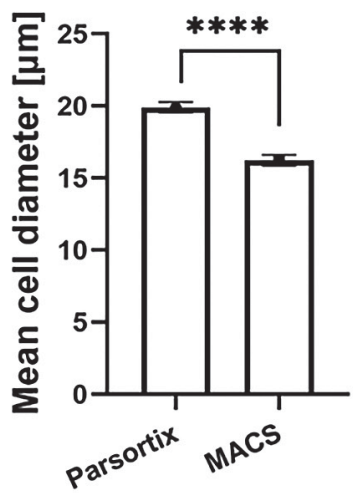

(E)

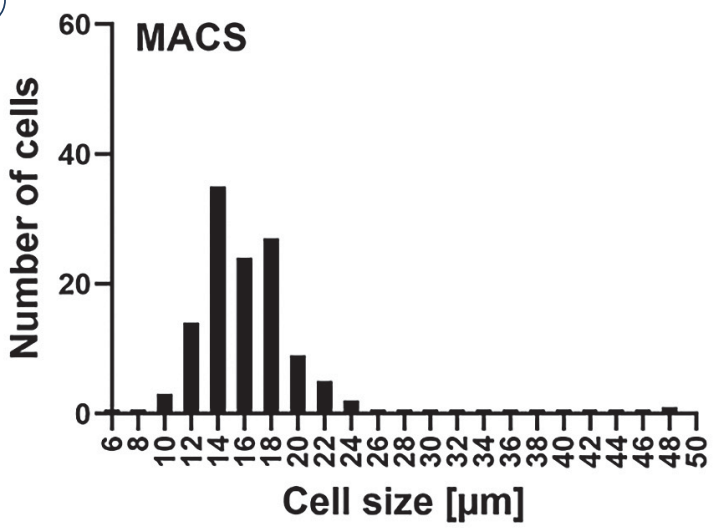

FIGURE 4. Parsortix® separation enabled identification of more CTCS and their size was larger compared to MACS $\AA^{\circledR}$ separation. (A) Total number of identified CTCs in the patient cohort. (B) Number of identified CTCs for each individual patient after Parsortix ${ }^{B}$ or MACS® separation. (C) Mean cell diameter of CTCS after Parsortix® or MACS® separation. (D) Histogram showing CTC size distribution after Parsortix ${ }^{\circledR}$ separation and $(\mathrm{E})$ MACS $\AA$ separation.

$* * * *=0.0001 ; n=$ total number of cells

blood collected through the same blood draw. CTCs were identified and enumerated based on their cytomorphological features such as their size (CTCs larger than $6.5 \mu \mathrm{m}$ ), round or oval shape, large nucleus, scant cytoplasm and a high $\mathrm{N} / \mathrm{C}$ ratio. In some cases, mitoses were observed which were suggestive of CTCs since mitosis is not typically observed in normal blood cells. After Parsortix $\AA$ separation, higher numbers of CTCs were identified. In total, Parsortix ${ }^{\circledR}$ separation resulted in identification of 221 CTCs and MACS® separation in 120 CTCs altogether (Figure 4A). In $58 \%$ of the patients, more CTCs were identified after Parsortix® separation, in $27 \%$ of patients more CTCs were identified after MACS $®$ separation and in $15 \%$ equal numbers of CTCs were identified after both separation techniques (Figure 4B). The identified CTCs were diverse in their size. CTC mean diameter was significantly larger in CTCs after
Parsortix ${ }^{\circledR}$ separation (Figure $4 \mathrm{C}$ ). Histograms of CTC sizes demonstrating that smaller CTCs were identified after MACS® separation are presented in Figure 4D and Figure 4E.

Besides cell size, different separation techniques also affected the morphology of CTCs. The identified CTCs were classified as morphologically "preserved" and "unpreserved" based on their morphological characteristics. After Parsortix ${ }^{\circledR}$ separation, the majority of identified CTCs were presented with preserved morphology with only 2 CTCs being identified as unpreserved (Figure 5A). Contrary, the majority of CTCs after MACS® separation were presented with unpreserved morphology, only one preserved CTC was identified after this separation technique (Figure 5B). The preserved CTCs were presented as cells with morphological features of malignancy and blast morphology such as large nuclei, high N/C ratio, 
scant basophilic cytoplasm and finely granular chromatin structure (Figure 5C). Nucleoli were not visible. CTCs also presented signs of degeneration such as cytoplasmic blebs and cytoplasmic villous projections (Figure 5C). Some of the cells were in a state of active mitosis, which is also a characteristic of tumor cells (Figure 5C). After Parsortix ${ }^{\circledR}$ separation routine cytokeratin AE1/AE3 (CK) immunocytochemical staining was positive in 2 out of 30 patients that were morphologically identified as preserved CTCs (Figure 5C, CK). Vimentin staining was not confirmed in any of the samples due to the suboptimal ICC reaction. The external controls displayed a strong positive reaction, however internal controls (lymphocytes) did not stain properly. Therefore, the staining was not considered valid and further optimization steps are warranted. Morphologically unpreserved CTCs were presented as cells with morphological features of malignancy such as scant eosinophilic cytoplasm with vacuoles and eosinophilic inclusions, large nuclei, high N/C ratio, and irregular nuclear contours with loss of chromatin structure (Figure 5C). After MACS $®$ isolation, unpreserved CTCs were positive for routine cytokeratin AE1/AE3 staining in 2 out of 30 patients.

\section{CTC polymorphism in metastatic breast cancer is dependent on the isolation protocol}

In this metastatic breast cancer patient cohort, highly polymorphic CTCs with blast morphology were identified. CTCs isolated by Parsortix ${ }^{\circledR}$ from the same blood sample varied in size (Figure 6). Numerous CTCs presented villous projections or cytoplasmic blebs, however, plasma membrane of some CTCs was smooth (Figure 6). Cytoplasm was predominantly scant and in some cells, it was almost not visible (Figure 6). MACS® separation from a parallel blood sample collected in the same blood draw resulted in predominantly unpreserved CTCs that also varied in size (Figure 6). Their size was generally smaller (Figure $4 \mathrm{C}$ and Figure 6), sometimes the size of a lymphocyte.

\section{Discussion}

In the preclinical part of study, we demonstrated that Parsortix ${ }^{\circledR}$ and MACS $®$ isolation techniques retained the viability and antigenic characteristics of MCF7 breast cancer cells. Hydropic and some other signs of degeneration such as cytoplasmic
TABLE 1. Patient characteristics

\begin{tabular}{|c|c|}
\hline Characteristics & Frequency $\mathrm{N}(\%)$ \\
\hline \multicolumn{2}{|l|}{ Histology } \\
\hline Invasive ductal carcinoma & $28(93.3)$ \\
\hline Invasive lobular carcinoma & $2(6.7)$ \\
\hline \multicolumn{2}{|l|}{ Tumor stage } \\
\hline $\mathrm{Tl}$ & $5(16.7)$ \\
\hline $\mathrm{T} 2$ & $18(60.0)$ \\
\hline T3 & $7(23.3)$ \\
\hline \multicolumn{2}{|l|}{$\mathrm{N}$ stage } \\
\hline NO & $5(16.7)$ \\
\hline NI & $9(30.0)$ \\
\hline N2 & $3(10.0)$ \\
\hline N3 & $7(23.3)$ \\
\hline Unknown & $6(20.0)$ \\
\hline \multicolumn{2}{|l|}{ Grade } \\
\hline Grade I & $1(3.3)$ \\
\hline Grade ॥ & $9(30.0)$ \\
\hline Grade III & $19(63.3)$ \\
\hline Unknown & $1(3.3)$ \\
\hline \multicolumn{2}{|l|}{ Hormone receptor } \\
\hline Estrogene receptor positive & $24(80.0)$ \\
\hline Estrogene receptor negative & $6(20.0)$ \\
\hline Progesterone receptor positive & $21(70.0)$ \\
\hline Progesterone receptor negative & $9(30.0)$ \\
\hline \multicolumn{2}{|l|}{ HER2 status } \\
\hline Positive & $4(13.3)$ \\
\hline Negative & $26(86.7)$ \\
\hline \multicolumn{2}{|l|}{ Molecular subtype } \\
\hline Luminal A-like & $8(25.8)$ \\
\hline Luminal-B like HER2 negative & $13(43.3)$ \\
\hline Luminal-B like HER2 positive & $3(10.0)$ \\
\hline HER2 positive & $1(3.3)$ \\
\hline Triple negative & $5(16.7)$ \\
\hline
\end{tabular}

blebs, villous projections and vacuolization were observed after separation with both techniques, however these changes were not severe, and the cells retained their viability. Parsortix ${ }^{\circledR}$ separation induced lower levels of degeneration compared to MACS $®$ in some of the evaluated morphological features. MACS® samples also contained high background of remaining hematopoietic cells. In the clinical setting in metastatic breast cancer patient cohort, we demonstrated that morphologically preserved CTCs were detected by Parsortix ${ }^{\circledR}$ method only. On the contrary, after MACS® separation the majority of detected CTCs were morphologically unpreserved. Furthermore, we demonstrate that CTCs from metastatic breast cancer show a high degree of polymorphism, even within the same patient. Taken together, morphological features of isolated CTCs are dependent on the separation technique and also to some degree to the in vivo degeneration in the blood stream. Parsortix ${ }^{\circledR}$ separation was demonstrated to be a 
suitable technique for detection of CTCs with preserved cell morphology in metastatic breast cancer for their identification by routine cytopathological techniques.

In our metastatic breast cancer patient cohort, Parsortix ${ }^{\circledR}$ separation was in general superior to MACS $®$ in terms of the quantity and quality of identified CTCs. However, in some samples, MACS samples contained higher numbers of CTCs. When comparing both methods, it should be kept in mind that the successful CTC isolation is not dependent only on the separation technique, but also on the blood sample. In our case, both blood samples were taken during the same blood draw, however in two separate $10 \mathrm{ml}$ blood collection tubes. Therefore, due to the fast blood flow it cannot be expected that both samples will contain equal numbers of CTCs, as CTC numbers in blood can depend on dynamics of CTC shedding from the tumor and also their distribution in the blood stream. Similar discrepancies in CTC numbers were observed also in the study of $\mathrm{Xu}$ et al., comparing three isolation platforms. ${ }^{20}$

Higher number of identified CTCs by Parsortix ${ }^{\circledR}$ separation can be to some extent attributed to its higher sensitivity. The sizes of CTCs isolated in our study were all larger than $6.5 \mu \mathrm{m}$ (gap size in the Parsortix ${ }^{\circledR}$ cassette), therefore the Parsortix ${ }^{\circledR}$ isolation capacity was retained. High recovery rates of different cell lines were reported also when directly compared to EpCAM positive selection techniques. ${ }^{9,18,21}$ In the study of Maertens et al., the median recovery rate of the Parsortix ${ }^{\circledR}$ system was $66 \%$ compared to $23 \%$ for EpCAM based separation (Epithelial Enrich Dynabeads, Invitrogen) after spiking of different cell lines. ${ }^{21}$ The advantage of Parsortix ${ }^{\circledR}$ size exclusion separation is also that it enables the capture of CTCs of different phenotypes, therefore higher capture capacity can be expected. Parsortix $\AA$ separation was demonstrated to identify significantly more mesenchymal human CTCs compared to the clinical CellSearch system. ${ }^{22}$ Contrary, MACS $\AA$ separation is limited by the capture of CTCs of epithelial phenotype, as was already confirmed in our previous study in spiking experiments of human fibroblasts into the buffy coat, demonstrating $0 \%$ recovery rate for mesenchymal cell line. ${ }^{8}$ CTCs found in the blood of cancer patients are a heterogeneous population as epithelial, mesenchymal and hybrid E/M phenotypes were detected in different cancer types. ${ }^{23-25}$ Detection of mesenchymal phenotype was shown to be associated with distant metastasis in breast cancer patients, therefore detection of CTCs with
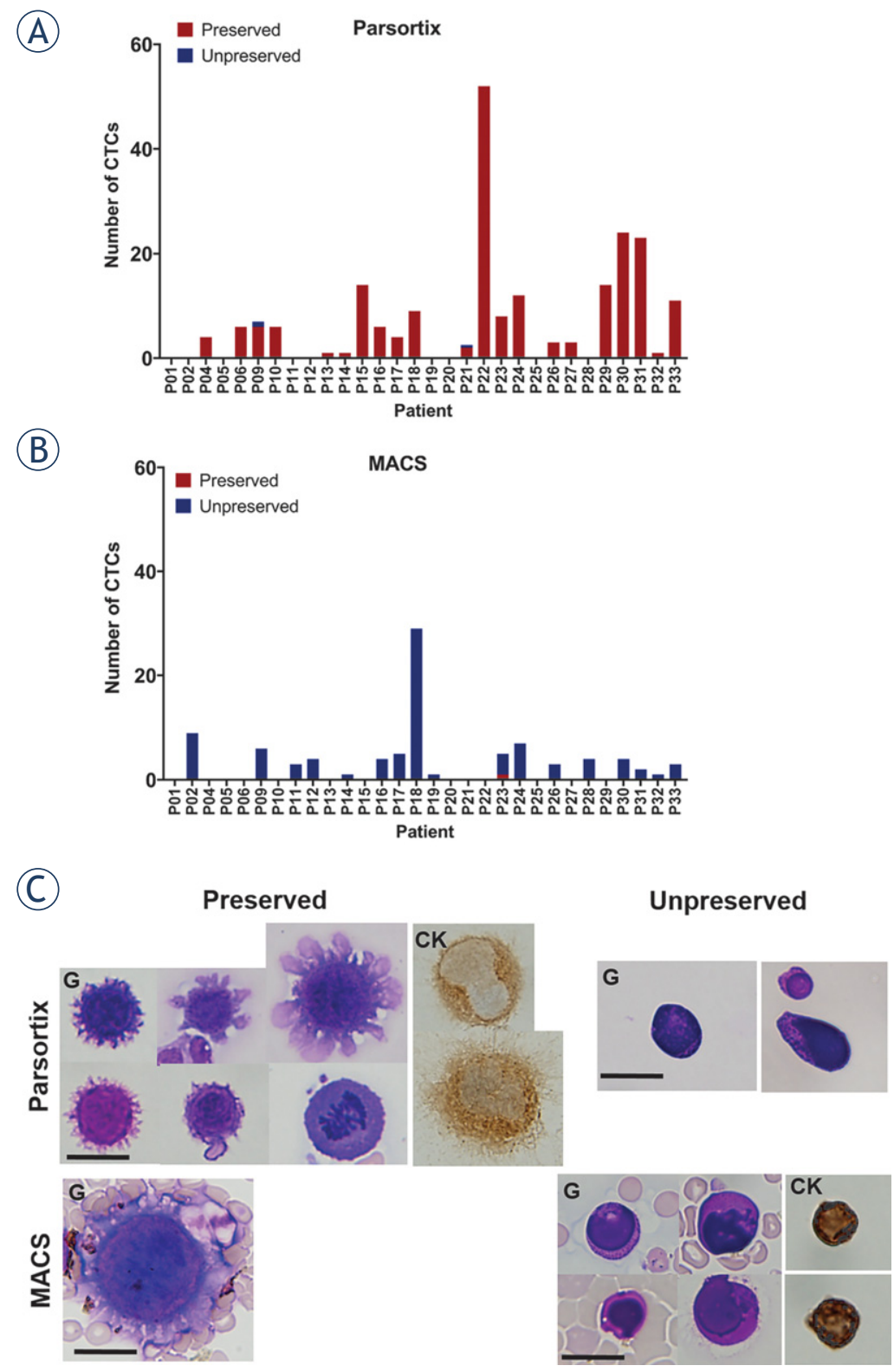

FIGURE 5. Effect of separation techniques on morphology of breast cancer CTCs. (A) Number of morphologically preserved or unpreserved CTCs after Parsortix ${ }^{\circledR}$ separation. (B) Number of morphologically preserved or unpreserved CTCs after MACS $\AA$ separation. (C) Images of CTCS with preserved or unpreserved morphology after both separation techniques. Scale bar represents $20 \mu \mathrm{m}$. Cells were stained with Giemsa (G) and cytokeratin AE1/AE3 (CK).

this phenotype seems to be of outmost importance. ${ }^{24}$ In our study we detected only 2 out of 30 patients with advanced metastatic disease with epithelial phenotype. CTCs presented with blast morphology, however unfortunately we could not confirm their mesenchymal phenotype due to the problems with our routine vimentin ICC staining, which seems to be suboptimal for CTC slides ob- 

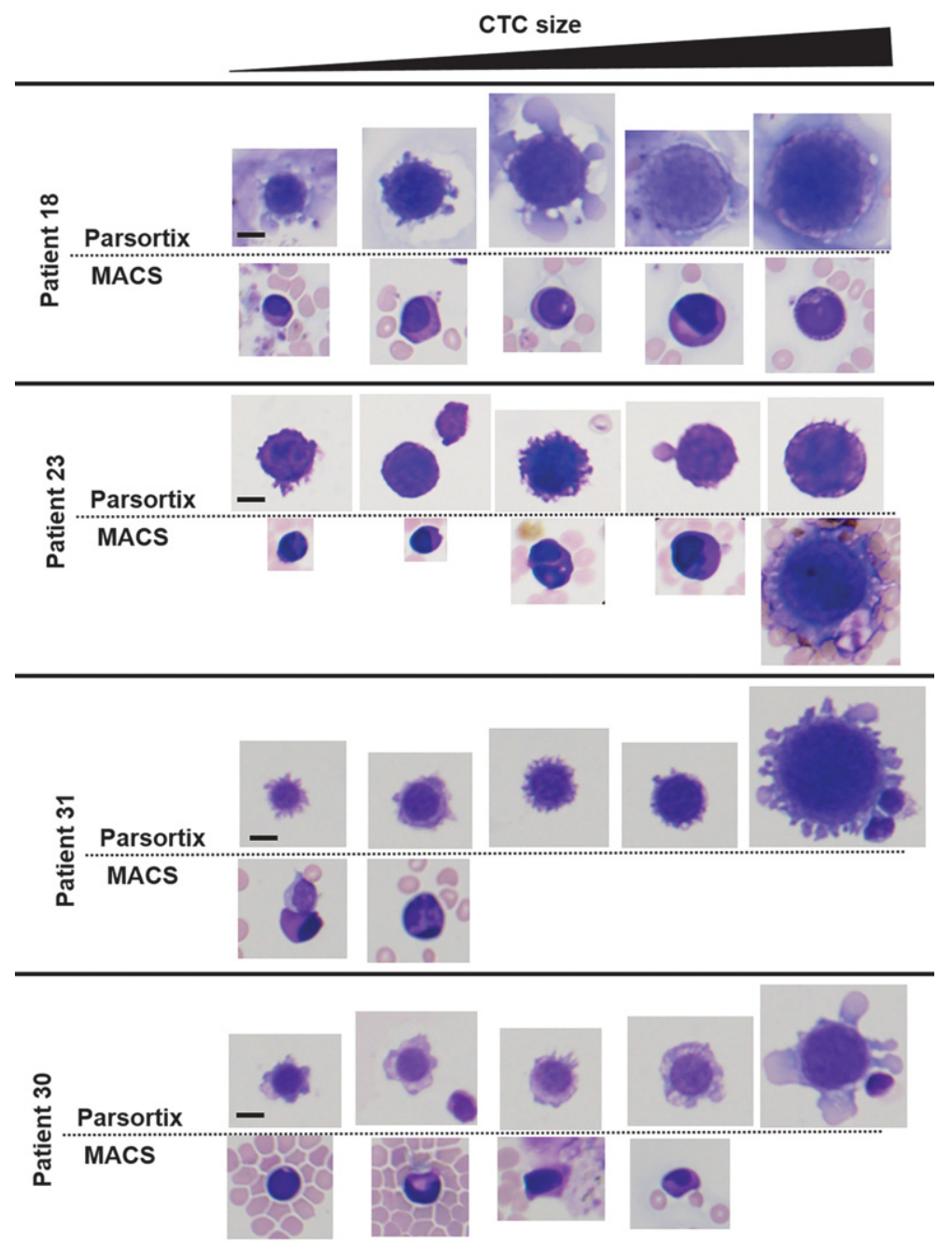

FIGURE 6. Morphological polymorphism of CTCs. Images of identified CTCs on a single patient level in four patients (patient 18, 23, 30, 31) are presented following Parsortix $\AA^{\circledR}$ or MACS $\AA$ separation from blood obtained in a single blood draw. CTCs varied in size and their morphological characteristics. Scale bar represents $10 \mu \mathrm{m}$.

tained in our study protocol. Further optimization steps for fixation and ICC staining are warranted.

Parsortix $®$ separation was demonstrated to preserve the morphological properties of CTCs much better than MACS $\AA$ separation. Both blood samples were taken during the same blood draw in the same type of collection tube and both separations were started at the same time, therefore the CTCs were retained at the same environmental conditions until the separation. Separation that followed was different. Parsortix ${ }^{\circledR}$ separation is based on microfluidic system that retain larger cells in the cassette with $6.5 \mu \mathrm{m}$ size gap. In MACS® separation, blood is passed through a dense magnetic column and cells are retained by a strong magnetic field. After both separations, cells were harvested and cytological slides were prepared by the same protocol. For Parsortix $®$, harvest (elution) was performed directly from the instrument into the in-house washing buffer. For MACS $₫$, elution of cells from the column was made by MACS® Whole Blood Column Elution Buffer of undisclosed composition. Therefore, the differences in cytomorphology of separated cells can arise from the separation technique itself and also the use of different recommended buffers for separation and elution.

A majority of patient' CTCs presented with the signs of morphological degeneration such as cytoplasmic blebs and villous projections after Parsortix ${ }^{\circledR}$ separation. Compared to our preclinical morphological evaluation of MCF7 cells, CTCs isolated from patient samples were more degenerated, displayed loss of chromatin structure with invisible nucleoli. The higher level of degeneration could be attributed to in vivo degeneration in the blood stream. After MACS® separation, degeneration was more severe and the cells displayed the characteristics of cell death such as loss of plasma membrane and nuclear membrane integrity, loss of chromatin structure, vacuoles and cytoplasmic eosinophilic inclusions. CTCs with the similar morphological characteristics were identified in our previous study in early breast cancer patient cohort and were presented as canonical and noncanonical. ${ }^{8}$ CTCs were termed canonical if malignant morphological features as well as cytokeratin positivity were observed and non-canonical if malignant morphological features were observed but there was no cytokeratin expression. ${ }^{8}$ In the current study, we were unable to use the same criteria as Parsortix ${ }^{\circledR}$ separation enables the isolation of CTCs with various phenotypes, therefore cytokeratin positive staining could not be considered as an identification criterion. Therefore, we focused exclusively on morphological features of cells, which are still a gold standard in cytopathology. ${ }^{26}$ For more precise characterization of CTCs further immunochemical studies of their phenotype are required. Our routine cytokeratin AE1/ AE3 staining resulted in positive staining in 2 out of 30 patients and routine vimentin staining was not positive in neither of the patients. Despite the fact that this staining performs well in the routine work, the staining is most probably not optimal for the CTCs from clinical samples as the signal of the staining was poor. Same was confirmed for vimentin staining, which could not be properly detected. The external controls displayed positive reaction, however internal controls (lymphocytes) did not 
stain properly, therefore the staining was not considered valid. Therefore, we believe that optimization of the routine immunocytochemical staining of CTCs is needed in order to stain these delicate group of cells. Therefore, our next study is aimed at optimization of immunocytochemical and immunofluorescent staining protocols for breast cancer CTCs taking in mind the distinct phenotypes and other markers used for identification in breast cancer cells.

The discrepancy in cytomorphological changes after both separation techniques in preclinical in clinical setting could imply on the fact that patient' CTCs are more sensitive and susceptible to degeneration as cell lines. CTCs in the blood are exposed to the shear stress of blood flow, lack of growth factors and immune surveillance, which can degenerate a large proportion of CTCs. ${ }^{10}$ These in vivo degenerated CTCs can also be more susceptible to subsequent stress during the separation. Furthermore, in our protocol, their degeneration can be enhanced also by centrifugation in a cytocentrifuge when preparing the cytological slides.

To the best of our knowledge, our study is one of the few studies investigating detailed morphological features of breast cancer CTCs with routine cytopathological techniques. A direct comparison to other studies of CTC morphology in distinct cancer types cannot be made as the protocols for separation and visualization are different. Therefore, a comparison can be made only based on the published images. In the study of Hattori et al. in breast cancer and Tsutsuyama et al. in colorectal patients, morphologically preserved single or clustered CTCs were obtained. ${ }^{14,15}$ The cells showed no sign of hydropic degeneration and the nuclear structure was preserved, as the samples were fixed in formalin prior to cytological slides preparation. Both studies utilized Optnics Precision Co. filtration device, which therefore also seems suitable for cytology-based detection of CTCs. In a case study of Marrinnuci et al., 659 CTCs were identified in a single $10 \mathrm{ml}$ blood sample in metastatic breast cancer patient following the fiber-optic array scanning technology (FAST) cytometry. ${ }^{13}$ Similar to our study, they found that the patient's CTCs exhibit a high degree of polymorphism with CTCs exhibiting early and late apoptotic changes..$^{13}$ In the study of Kuvendjiska et al. in non-metastatic esophageal adenocarcinoma, different types of CTCs were identified based on their morphological features after ISET filtration. ${ }^{16}$ Small and large single-CTCs, cluster CTCs and circulating cancer-associated macrophage-like cells (CAML) were identified.
CTCs presented with increased N/C ratio, enlarged and hyperchromatic nuclei, loss of chromatin structure, irregular nuclear borders and sometimes multilobulated nuclei, therefore, their morphology was to our opinion not sufficiently preserved. ${ }^{16}$

This study was a preliminary study, aimed at side-by-side comparison of two available separation techniques in order to identify which of the two methods is more appropriate for integration into our routine clinical cytopathology laboratory. The integration of this method to our laboratory will allow us to conduct subsequent clinical studies, which will be aimed at investigation of the clinical relevance of the CTC number and their preserved morphology in different tumor types.

In conclusion, Parsortix ${ }^{\circledR}$ technology is straightforward technology for CTC isolation, which enables the preservation of cell morphology and can be easily integrated into a routine cytopathology laboratory. Cytopathological analysis and microscopic examination of cells is still the gold standard in cytopathology and histology in cancer management. The advantage of cytopathological analysis is that it is easily accessible in the clinical environment as cytopathologists are an integral part of cancer patient care. Therefore, the analysis can be done without the use of special techniques and instruments such as flow cytometry or single cell next generation sequencing (NGS), which require substantial financial and human resources.

\section{Acknowledgments}

We would like to thank all patients participating in this study. We would also like to thank ANGLE plc. for providing the Parsortix ${ }^{\circledR}$ instrument for evaluation in our lab with special thanks to Adam Giles, Michael O'Bri-en and Lara Stevenato for a detailed training and their valuable advice. We acknowledge the financial support of Slovenian Research Agency (grant no. P3-0003, P3-0289 and P3-0321).

\section{References}

1. Bilani N, Elson L, Liang H, Elimimian EB, Arteta-Bulos R, Nahleh Z. Prognostic and predictive value of circulating and disseminated tumor cells in breast cancer: A National Cancer Database (NCDB) analysis. Technol Cancer Res Treat 2020; 19: 1533033820980107. doi: 10.1177/1533033820980107

2. Theil G, Fornara P, Bialek J. Position of circulating tumor cells in the clinical routine in prostate cancer and breast cancer patients. Cancers 2020; 12: 3782. doi: $10.3390 /$ cancers 12123782

3. Lozar T, Gersak K, Cemazar M, Grasic Kuhar C, Jesenko T. The biology and clinical potential of circulating tumor cells. Radiol Oncol 2019; 53: 131-47. doi: 10.2478/raon-2019-0024 
4. Grosse-Wilde A, Fouquier d'Hérouël A, McIntosh E, Ertaylan G, Skupin A Kuestner RE, et al. Stemness of the hybrid epithelial/mesenchymal state in breast cancer and its association with poor survival. PLoS One 2015; 10: e0126522. doi: 10.1371/journal.pone.0126522

5. Jolly MK, Boareto M, Huang B, Jia D, Lu M, Ben-Jacob E, et al. Implications of the hybrid epithelial/mesenchymal phenotype in metastasis. Front Oncol 2015; 5:155. doi: 10.3389/fonc.2015.00155

6. Riethdorf S, Fritsche H, Muller V, Rau T, Schindlbeck C, Rack B, et al. Detection of circulating tumor cells in peripheral blood of patients with metastatic breast cancer: a validation study of the CellSearch System. Clin Cancer Res 2007; 13: 920-8. doi: 10.1158/1078-0432.CCR-06-1695

7. Hu XC, Wang Y, Shi DR, Loo TY, Chow LWC. Immunomagnetic tumor cell enrichment is promising in detecting circulating breast cancer cells. Oncology 2003; 64: 160-5. doi: 10.1159/000067776

8. Lozar T, Jesenko T, Kloboves Prevodnik V, Cemazar M, Hosta V, Jericevic A, et al. Preclinical and clinical evaluation of magnetic-activated cell separation technology for CTC isolation in breast cancer. Front Oncol 2020; 10: 554554. doi: $10.3389 /$ fonc. 2020.554554

9. Miller MC, Robinson PS, Wagner C, O'Shannessy DJ. The Parsortix ${ }^{\mathrm{TM}}$ cell separation system - a versatile liquid biopsy platform. Cytometry A 2018; 93: 1234-9. doi: 10.1002/cyto.a.23571

10. Huang $Q, H u X, H e ~ W, Z$ hao $Y, H a o ~ S, W u ~ Q$, et al. Fluid shear stress and tumor metastasis. Am J Cancer Res 2018; 8: 763-777. PMID: 29888101

11. Lowe AC, Pignon JC, Carvo I, Drage MG, Constantine NM, Jones N, et al. Young investigator challenge: application of cytologic techniques to circulating tumor cell specimens: detecting activation of the oncogenic transcription factor STAT3. Cancer Cytopathol 2015; 123: 696-705. doi: 10.1002/ cncy. 21640

12. Kamal M, Macall L, Horton C, Hills N, Davis R, Nguyen R, et al. Cytopathologic identification of circulating tumor cells (CTCS) in breast cancer: Application of size-based enrichment. Clin Diagnostic Pathol 2019; 3: 1-5. doi: 10.15761/ CDP.1000S1001

13. Marrinucci D, Bethel K, Bruce RH, Curry DN, Hsieh B, Humphrey M, et al. Case study of the morphologic variation of circulating tumor cells. Hum Pathol 2007; 38: 514-9. doi: 10.1016/j.humpath.2006.08.027

14. Hattori $M$, Nakanishi $H$, Yoshimura $M$, Iwase $M$, Yoshimura $A$, Adachi $Y$, et al. Circulating tumor cells detection in tumor draining vein of breast cancer patients. Sci Rep 2019; 9: 18195. doi: 10.1038/s41598-019-54839-y

15. Tsutsuyama M, Nakanishi $H$, Yoshimura M, Oshiro T, Kinoshita T, Komor $\mathrm{K}$, et al. Detection of circulating tumor cells in drainage venous blood from colorectal cancer patients using a new filtration and cytology-based automated platform. PLoS One 2019; 14: e0212221. doi: 10.1371/journal. pone.0212221

16. Kuvendjiska J, Pitman MB, Martini V, Braun C, Grebe K, Timme S, et al. Cytopathological heterogeneity of circulating tumor cells in non-metastatic esophageal adenocarcinoma. Anticancer Res 2020; 40: 5679-85. doi 10.21873/anticanres.14582

17. Lee AV, Oesterreich S, Davidson NE. MCF-7 cells - changing the course of breast cancer research and care for 45 years. J Natl Cancer Inst 2015; 107 djv073. doi: 10.1093/jnci/djv073

18. Koch C, Joosse SA, Schneegans S, Wilken OJW, Janning M, Loreth $\mathrm{D}$, et al. Pre-analytical and analytical variables of label-independent enrichment and automated detection of circulating tumor cells in cancer patients. Cancers 2020; 12: 442. doi: 10.3390/cancers 12020442

19. Schneider CA, Rasband WS, Eliceiri KW. NIH Image to ImageJ: 25 years of image analysis. Nature Methods 2012; 9: 671-5. doi: 10.1038/nmeth.2089

20. Xu L, Mao X, Imrali A, Syed F, Mutsvangwa K, Berney D, et al. Optimization and evaluation of a novel size based circulating tumor cell isolation system. PLoS One 2015; 10: e0138032. doi: 10.1371/journal.pone.0138032

21. Maertens $Y$, Humberg V, Erlmeier F, Steffens $S$, Steinestel J, Bögemann $M$, et al. Comparison of isolation platforms for detection of circulating renal cell carcinoma cells. Oncotarget, 2017; 8: 87710-7. doi: 10.18632/oncotarget.21197

22. Kitz J, Goodale D, Postenka C, Lowes LE, Allan AL. EMT-independent detection of circulating tumor cells in human blood samples and preclinical mouse models of metastasis. Clin Exp Metastasis 2021; 38: 97-108. doi:10.1007/s10585-020-10070-y.
23. Pan L, Yan G, Chen W, Sun L, Wang J, Yang J. Distribution of circulating tumor cell phenotype in early cervical cancer. Cancer Manag Res 2019; 11: 5531-5536. doi: 10.2147/CMAR.S19839.

24. Zhang S, Wu T, Peng X, Liu J, Liu F, Wu S, et al. Mesenchymal phenotype of circulating tumor cells is associated with distant metastasis in breast cancer patients. Cancer Manag Res 2017; 9: 691-700. doi: 10.2147/CMAR. S149801

25. Messaritakis I, Politaki E, Kotsakis A, Dermitzaki EK, Koinis F, Lagoudaki E, et al. Phenotypic characterization of circulating tumor cells in the peripheral blood of patients with small cell lung cancer. PLoS One 2017; 12: e0181211. doi: 10.1371/journal.pone.0181211

26. Dey P. Routine staining in cytology laboratory. In: Basic and advanced laboratory techniques in histopathology and cytology. Singapore: Springer; 2018. p. $133-8$. 